Supporting Information

\title{
Enzyme-like Kinetics of Ferryloxy Myoglobin Formation in Films on
}

\section{Electrodes in Microemulsions}

Peterson M Guto ${ }^{l}$ and James F. Rusling ${ }^{1,2}$

Contribution from ${ }^{1}$ Department of Chemistry, University of Connecticut, Storrs, Connecticut 06269-3060, and ${ }^{2}$ Department of Pharmacology, University of Connecticut Health Center, Farmington, Connecticut 06032,Email: James.Rusling@uconn.edu

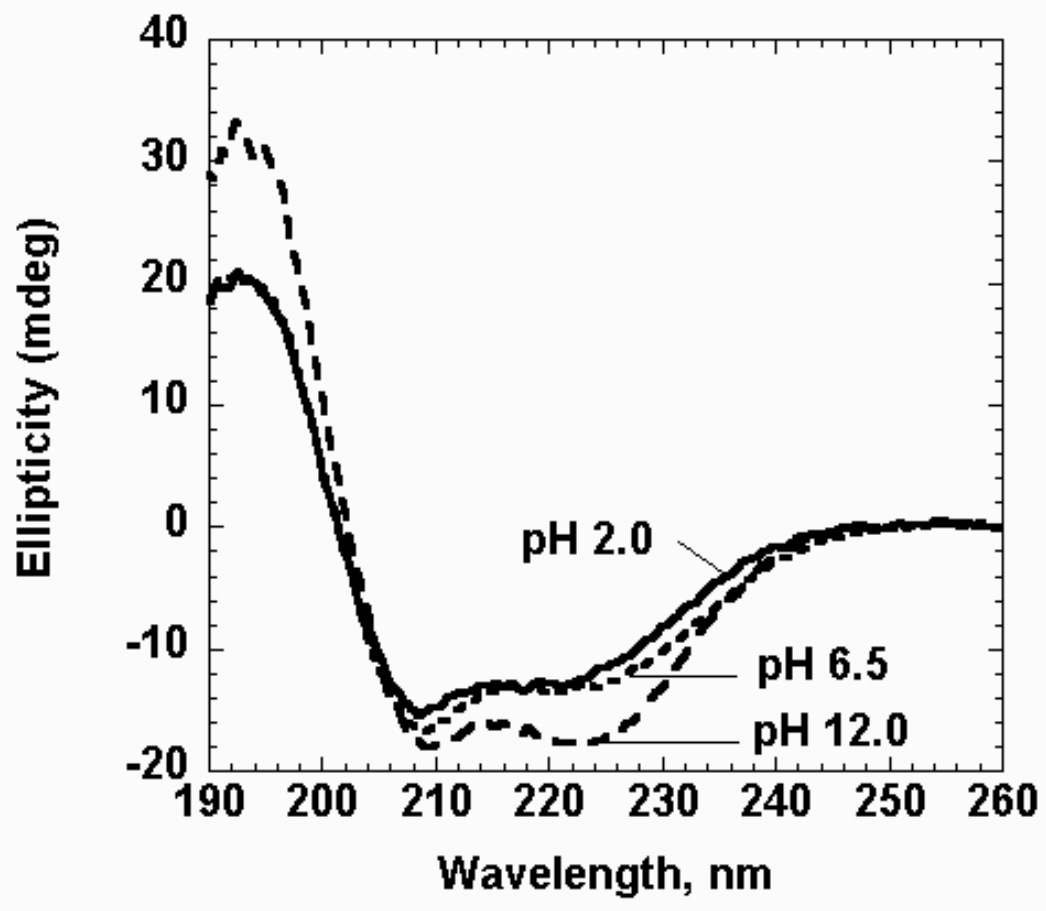

Figure S1: CD spectra of myoglobin in PLL/Mb films in phosphate buffer solutions with varying $\mathrm{pH}$. The specific $\mathrm{pH}$ values are indicated on respective spectrum. Backgrounds from control PLL films without Mb have been subtracted. The PLL background spectra were featureless. 


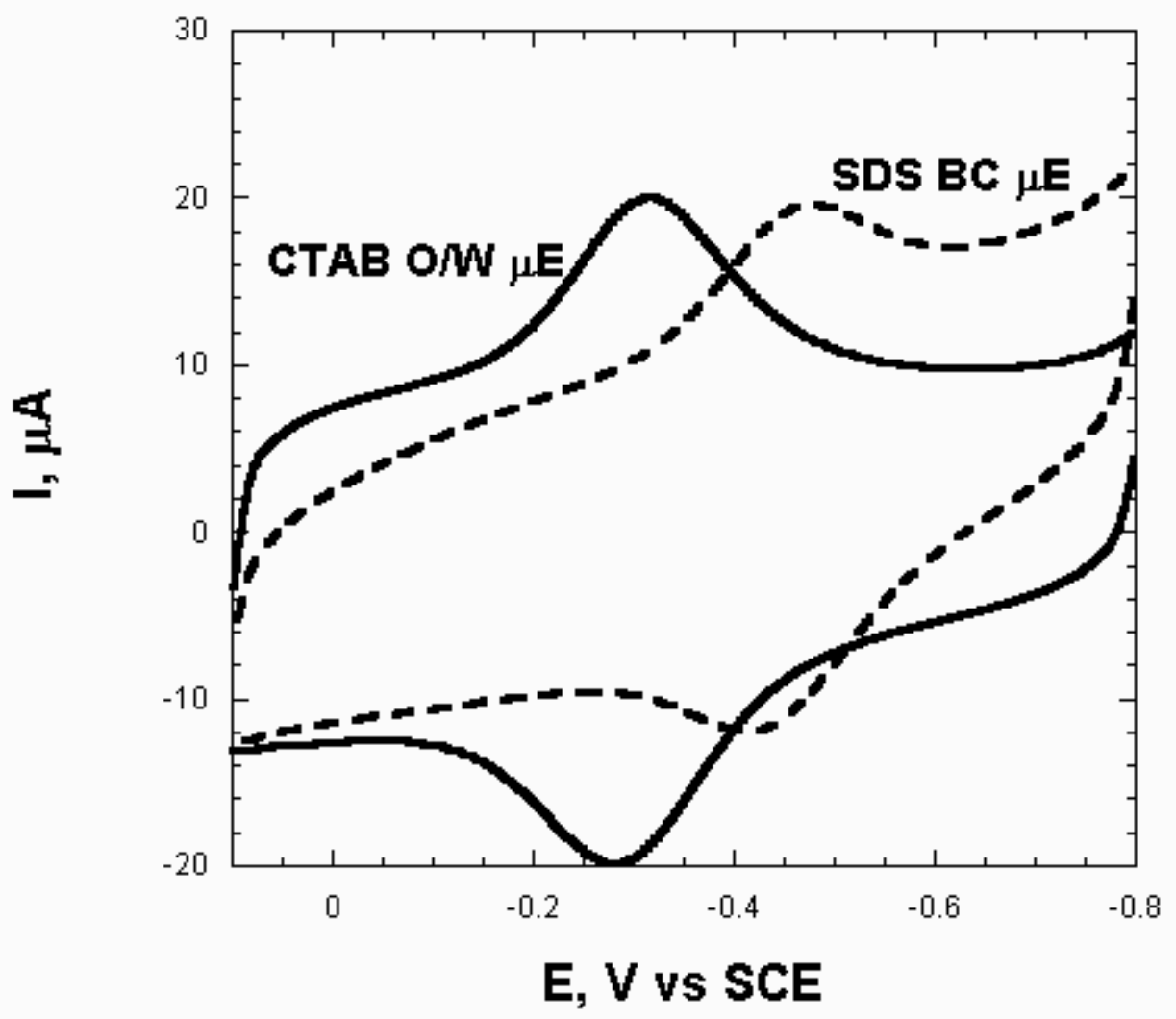

Figure S2: Cyclic voltammograms of the PLL/Mb films in CTAB oil/water microemulsion $(\mu \mathrm{E})$ (solid line) and SDS bicontinuous microemulsion $(\mu \mathrm{E})$ (dotted line) at $200 \mathrm{mV} \mathrm{s}^{-1}$. 


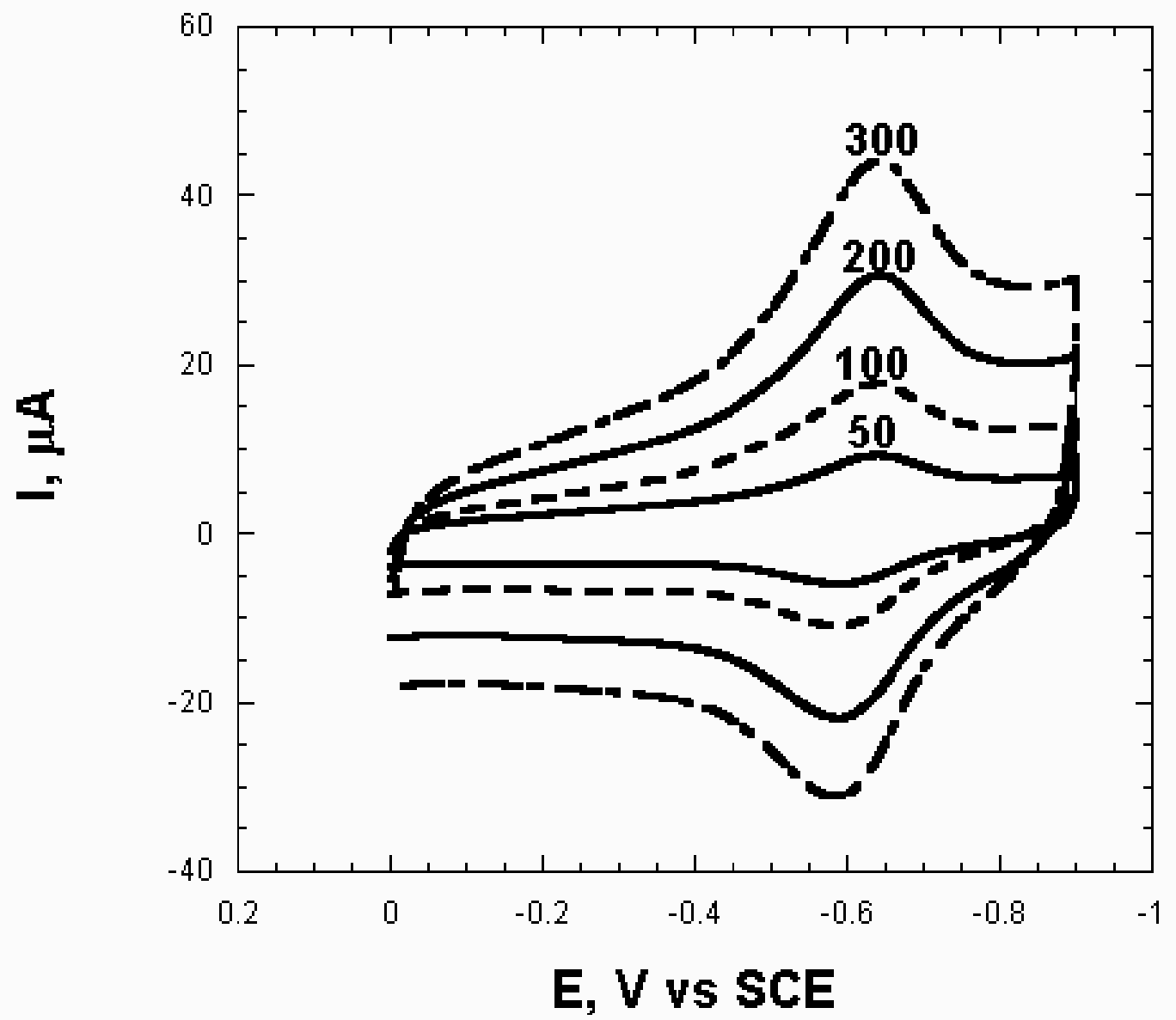

Figure S3: Cyclic voltammograms of PLL/Mb films in CTAB bicontinuous microemulsions of $\mathrm{pH} 12.0$ at scan rates of 50,100, 200 and $300 \mathrm{mV} \mathrm{s}^{-1}$. 


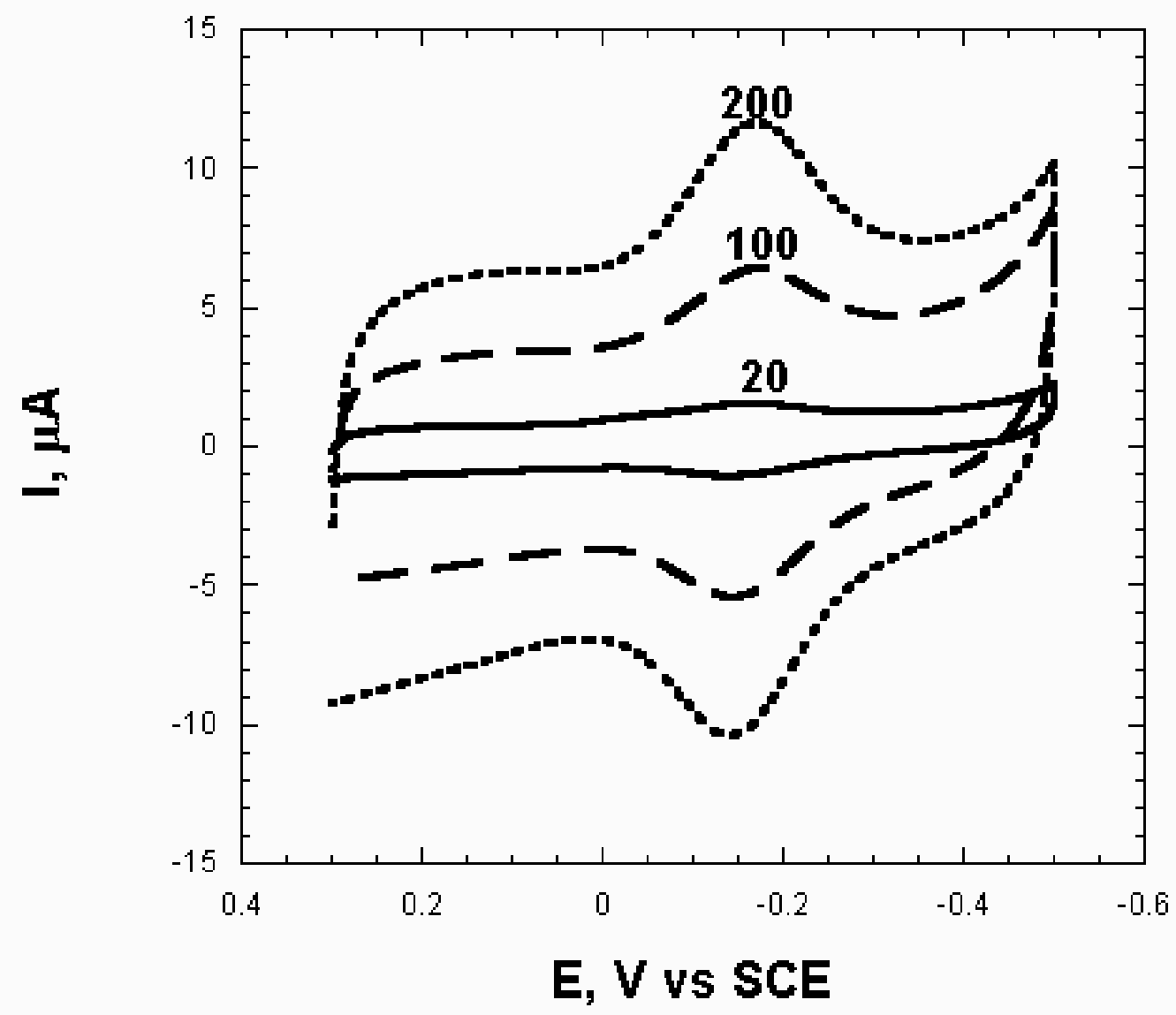

Figure S4: Cyclic voltammograms of PLL/Mb films in SDS oil in water microemulsions of $\mathrm{pH} 2.0$ at scan rates of 20,100 and $200 \mathrm{mV} \mathrm{s}^{-1}$. 
Table S1. Apparent Michaelis parameters $k_{\text {cat }}, K_{m}$ and $k_{c a t} / K_{m}$ from RDV for reduction of $\mathrm{tBuOOH}$ on $\mathrm{PLL} / \mathrm{Mb}$ films at $1000 \mathrm{rpm}$ in microemulsions from the concentration dependence for fluids with neutral water phases

\begin{tabular}{|c|c|c|c|}
\hline Media & $k_{\text {cat }}\left(s^{-1}\right)$ & $K_{m}(\mathbf{m M})$ & $10^{-4} k_{\text {cat }} / K_{m}\left(M^{-1} s^{-1}\right)$ \\
\hline $\mathrm{SDS} \mathrm{B/C}$ & $9.8 \pm 1.1$ & $0.74 \pm 0.05$ & $1.4 \pm 0.05$ \\
\hline $\mathrm{SDS} \mathrm{O} / \mathrm{W}$ & $14.3 \pm 1.2$ & $1.2 \pm 0.2$ & $1.2 \pm 0.15$ \\
\hline SDS micellar solution & $10.7 \pm 1.0$ & $0.50 \pm 0.14$ & $2.2 \pm 0.35$ \\
\hline $\mathrm{CTAB} \mathrm{B} / \mathrm{C}$ & $7.3 \pm 0.2$ & $0.63 \pm 0.03$ & $1.2 \pm 0.04$ \\
\hline $\mathrm{CTAB} \mathrm{O} / \mathrm{W}$ & $6.2 \pm 0.7$ & $0.59 \pm 0.13$ & $1.1 \pm 0.1$ \\
\hline CTAB micellar solution & $16.0 \pm 1.5$ & $0.62 \pm 0.05$ & $2.6 \pm 0.04$ \\
\hline Phosphate Buffer, PH 6.5 & $16.4 \pm 2.5$ & $0.17 \pm 0.03$ & $9.6 \pm 0.15$ \\
\hline
\end{tabular}


Table S2. Apparent Michaelis parameters $k_{\text {cat }}, K_{m}$ and $k_{c a t} / K_{m}$ from RDV for reduction of $\mathrm{tBuOOH}$ on $\mathrm{PLL} / \mathrm{Mb}$ films at $1000 \mathrm{rpm}$ in microemulsions from the concentration dependence for fluids with $\mathrm{pH} 2.0$ water phases

\begin{tabular}{lccc}
\hline Media & $\mathbf{k}_{\mathbf{c a t}}\left(\mathbf{s}^{\mathbf{- 1}}\right)$ & $\mathbf{K}_{\mathbf{m}}(\mathbf{m} \mathbf{M})$ & $\mathbf{1 0}^{-4} \mathbf{k}_{\mathbf{c a t}} / \mathbf{K}_{\mathbf{m}}\left(\mathbf{M}^{-1} \mathbf{s}^{\mathbf{- 1}}\right)$ \\
\hline SDS B/C & $3.3 \pm 0.3$ & $0.025 \pm 0.001$ & $13 \pm 1$ \\
SDS O/W & $2.1 \pm 0.1$ & $0.013 \pm 0.001$ & $16 \pm 1$ \\
SDS micellar solution & $4.8 \pm 0.4$ & $0.12 \pm 0.01$ & $4.1 \pm 0.2$ \\
CTAB B/C & $2.9 \pm 0.6$ & $0.11 \pm 0.02$ & $3.5 \pm 1.5$ \\
CTAB O/W & $7.2 \pm 1.7$ & $0.11 \pm 0.01$ & $6.5 \pm 0.2$ \\
CTAB micellar solution & $4.0 \pm 0.8$ & $0.18 \pm 0.04$ & $2.3 \pm 0.1$ \\
Phosphate Buffer, PH 2.0 & $5.3 \pm 0.2$ & $0.029 \pm 0.002$ & $19 \pm 1$ \\
\hline
\end{tabular}


Table S3. Apparent Michaelis parameters $k_{\text {cat }}, K_{m}$ and $k_{c a t} / K_{m}$ from RDV for reduction of tBuOOH by $\mathrm{PLL} / \mathrm{Mb}$ films at $1000 \mathrm{rpm}$ in microemulsions from the concentration dependence system for fluids with $\mathrm{pH} 12.0$ water phases

\begin{tabular}{|c|c|c|c|}
\hline Media & $\mathbf{k}_{\text {cat }}\left(\mathrm{s}^{-1}\right)$ & $K_{m}(\mathbf{m M})$ & $10^{-4} k_{\text {cat }} / K_{m}\left(M^{-1} s^{-1}\right)$ \\
\hline $\mathrm{SDS} B / \mathrm{C}$ & $8.0 \pm 0.7$ & $0.18 \pm 0.02$ & $4.3 \pm 0.1$ \\
\hline $\mathrm{SDS} \mathrm{O} / \mathrm{W}$ & $5.3 \pm 0.2$ & $0.20 \pm 0.02$ & $2.7 \pm 0.04$ \\
\hline SDS micellar solution & $4.6 \pm 0.6$ & $0.38 \pm 0.03$ & $1.20 \pm 0.04$ \\
\hline CTAB B/C & $2.8 \pm 0.8$ & $0.19 \pm 0.05$ & $1.6 \pm 0.8$ \\
\hline $\mathrm{CTAB} \mathrm{O} / \mathrm{W}$ & $5.4 \pm 0.1$ & $0.25 \pm 0.08$ & $2.2 \pm 0.2$ \\
\hline CTAB micellar solution & $3.6 \pm 1$ & $0.48 \pm 0.16$ & $0.75 \pm 0.05$ \\
\hline Phosphate Buffer PH 12.0 & $2.9 \pm 1$ & $0.17 \pm 0.03$ & $1.7 \pm 0.3$ \\
\hline
\end{tabular}

